\title{
A DLM/FD/IB method for simulating compound vesicle motion under creeping flow condition
}

\author{
Tsorng-Whay Pan $^{1}$, Shihai Zhao, Xiting Niu, Roland Glowinski \\ Department of Mathematics, University of Houston, Houston, Texas 77204, USA
}

\begin{abstract}
In this article we present first a new distributed Lagrange multiplier/fictitious domain (DLM/FD) method for simulating fluid-particle interaction in Stokes flow. A conjugate gradient method driven by both pressure and distributed Lagrange multiplier, called one-shot method, has been developed to solve the discrete Stokes problem while enforcing the rigid body motion within the region occupied by the particle. The methodology is validated by comparing the numerical results of a neutrally buoyant particle of either a circular or elliptic shape with the associated Jeffery's solutions. We have successively combined the above methodology with an immersed boundary (IB) method and an elastic membrane modeled by a spring network to simulate the dynamics of a compound vesicle. In simple shear flow under creeping flow condition, the results are consistent with those obtained in literature. In Poiseuille flow, the compound vesicle motion is dominated by the motion of the vesicle membrane as expected and stays in the central region of the channel.

keywords: Stokes flow; Neutrally buoyant particle; Compound vesicle; Distributed Lagrange multiplier/fictitious domain method; Immersed boundary method.
\end{abstract}

\section{Introduction}

The biological cell is a lipid bilayer membrane encapsulating the cellular content. To model the red blood cell (RBC), vesicle and inextensible vesicle enclosing homogeneous fluid are often used. However, for eukaryotic cells (except the mature RBCs), the influence of the internal structure on its dynamics and rheology is not yet fully understood. For example, the nucleus occupies $18 \%$ to $44 \%$ of the volume in human leukocytes [1] and affects leukocyte adhesion to vascular endothelium. Malaria infected RBCs (iRBCs) have reduced deformability and shape changed and can disrupt the microcirculation of blood flow $[2,3]$. To model those biological cells and to study the cell dynamics in fluid flow, for examples, a compound vesicle, which has a suspended rigid particle inside its membrane, immersed in a fluid has been considered in [4], and a bilamellar vesicle consisting of two vesicles has been used in [5]. In [4] and [5], the internal structure does trigger the transition from tank-trading to

\footnotetext{
${ }^{1}$ Corresponding author: e-mail: pan@math.uh.edu, tel.: 713-743-3448
} 
tumbling in shear flow in the absence of any viscosity mismatch. For studying the dynamics of iRBCs in fluid flow, Plasmodium falciparum (P. falciparum) parasites inside the RBC membrane are modeled as rigid particles (e.g., see $[6,7,8]$ ). In this article we perform a computational study of compound vesicle in simple shear flow by using a spring network to model the cell membrane and treating P. falciparum parasite inside cell membrane as a neutrally buoyant particle under the creeping flow condition. The computational methods combining such spring network model with an immersed boundary (IB) method for simulating the motion of RBCs in twodimensional shear flow and Poiseuille flows have been developed in [9, 10, 11]. For simulating particle-fluid interaction under the creeping flow condition, we have developed a new distributed Lagrange multiplier/fictitious domain (DLM/FD) formulation to combine the Stokes equations and the Euler-Newton's equations in a way closely related to those developed and tested in, e.g., $[12,13,14,15,16,17,18,19,20]$ for simulating the particle motion in fluid flow at the finite Reynolds numbers. In the DLM/FD approach, the entire fluid-particle domain is considered to be a fluid. The fluid inside the particle boundary must exhibit a rigid-body motion. This constraint is enforced using the distributed Lagrange multiplier, which represents the additional body force per unit volume needed to maintain the rigid-body motion inside the particle boundary, much like the pressure in incompressible fluid flow, whose gradient is the force required to maintain the constraint of incompressibility. A similar approach has been developed in [21] for particulate-Stokes flow via a different constraint inside the particle. We have validated the methodology by comparing the numerical results of particle motion in shear flow with the Jeffery's solutions. We then integrate both DLM/FD method and IB method to simulate the dynamics of a compound vesicle (vesicle with a neutrally buoyant particle inside) in shear flow and Poiseuille flow. In shear flow, the inclination angles of compound vesicle with different filling fractions are consistent with those obtained in [22]. In Poiseuille flow, the motion of a compound vesicle is dominated by the motion of vesicle membrane. After migrating from the initial position next the wall to the central region of the channel, the particle moves horizontally without crossing the streamlines as expected. When a compound vesicle interacts with seven vesicles modeled as healthy RBCs in a microchannel, they all migrate to the central region and stay there under the creeping condition. The context of the article is as follows: We discuss DLM/FD formulations and then the related numerical schemes in Section 2. In Section 3, we validate our methods by comparing numerical results of particle motion with the Jeffery's solutions. Then numerical results of a compound vesicle are presented. The conclusions are summarized in Section 4. 


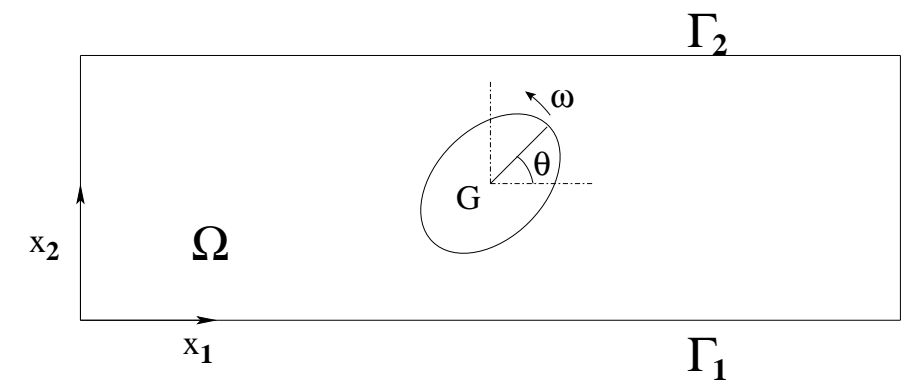

Figure 1: An example of flow region with one elliptic particle.

\section{Models and numerical methods}

\section{$2.1 \quad \mathrm{DLM} / \mathrm{FD}$ formulation}

Fictitious domain formulations with distributed Lagrange multiplier for flow around freely moving particles at finite Reynolds numbers and its associated computational methods have been developed and tested in, e.g., $[12,13,14,16]$. For the cases of neutrally buoyant particles, similar methodologies have been developed in $[15,17$, $18,19,20]$. But for the cases of a neutrally buoyant cylinder under creeping flow condition, similar DLM/FD method has not been discussed and fully validated yet. In this article, we discuss first the formulation for the case of an elliptic cylinder and then present the numerical results to validate the methodology. Let $\Omega \subset \mathbb{R}^{2}$ be a rectangular region filled with a Newtonian viscous incompressible fluid and containing a freely moving rigid particle $B$ centered at $\mathbf{G}=\left\{G_{1}, G_{2}\right\}^{t}$.

For the motion of fluid, the governing equations are the Stokes equations

$$
\begin{aligned}
& -\boldsymbol{\nabla} \cdot \boldsymbol{\sigma}=\rho_{f} \mathbf{g} \text { in }(\Omega \backslash \overline{B(t)}), t \in(0, T), \\
& \boldsymbol{\nabla} \cdot \mathbf{u}=0 \text { in }(\Omega \backslash \overline{B(t)}), t \in(0, T), \\
& \mathbf{u}=\mathbf{g}_{0} \text { on } \Gamma \times(0, T), \text { with } \int_{\Gamma} \mathbf{g}_{0} \cdot \mathbf{n} d \Gamma=0
\end{aligned}
$$

where $\mathbf{u}$ is the flow velocity, $\mathbf{g}$ is the gravity, $\boldsymbol{\sigma}=-p \mathbf{I}+2 \mu_{f} \mathbf{D}(\mathbf{u})$ with $2 \mathbf{D}(\mathbf{u})=$ $\boldsymbol{\nabla} \mathbf{u}+(\boldsymbol{\nabla} \mathbf{u})^{t}, p$ is the pressure, $\rho_{f}$ is the the density of fluid and $\mu_{f}$ is the viscosity of the fluid, $\Gamma$ is the union of the bottom boundary $\Gamma_{1}$ and top boundary $\Gamma_{2}$ as in Figure $1, \mathbf{n}$ is the unit normal vector pointing outward to the flow region, the boundary conditions are $\mathbf{g}_{0}=\{0,0\}^{t}$ when considering Poiseuille flow and $\mathbf{g}_{0}=\{-U / 2,0\}^{t}$ on $\Gamma_{1}$ and $\mathbf{g}_{0}=\{U / 2,0\}^{t}$ on $\Gamma_{2}$ when considering shear flow. We also assume that the flow is periodic in the $x_{1}$ direction with the period $L, L$ being the common length of the channel $\Omega$ and there is a no-slip condition on the boundary of the particle $\gamma(=\partial B(t))$

$$
\mathbf{u}(\mathbf{x}, t)=\mathbf{V}(t)+\omega(t) \overrightarrow{\mathbf{G x}}^{\perp}, \forall \mathbf{x} \in \partial B(t), t \in(0, T) .
$$


with $\overrightarrow{\mathbf{G x}}^{\perp}=\left\{-\left(x_{2}-G_{2}\right), x_{1}-G_{1}\right\}^{t}$. The motion of particle $B$ satisfies the EulerNewton's equations

$$
\begin{aligned}
& \mathbf{u}(\mathbf{x}, t)=\mathbf{V}(t)+\omega(t) \overrightarrow{\mathbf{G}}^{\perp}, \forall \mathbf{x} \in \partial B(t), t \in(0, T), \\
& \frac{d \mathbf{G}}{d t}=\mathbf{V}, \\
& \frac{d \theta}{d t}=\omega \\
& M_{p} \frac{d \mathbf{V}}{d t}=M_{p} \mathbf{g}+\mathbf{F}_{H}, \\
& I_{p} \frac{d \omega}{d t}=T_{H}, \\
& \mathbf{V}(0)=\mathbf{V}_{0}, \omega(0)=\omega_{0}, \mathbf{G}(0)=\mathbf{G}_{0}, \theta(0)=\theta_{0}
\end{aligned}
$$

where $M_{p}$ is the mass, $I_{p}$ is the inertia, $\mathbf{G}$ is the center of mass, $\mathbf{V}$ is the velocity of the center of mass, $\omega$ is the angular velocity of particle $B$ and $\theta$ is the inclination angle of the particle as in Figure 1. The hydrodynamical forces and torque are

$$
\mathbf{F}_{H}=-\int_{\gamma} \boldsymbol{\sigma} \mathbf{n} d \gamma, \quad T_{H}=-\int_{\gamma} \overrightarrow{\mathbf{G x}}^{\perp} \cdot \boldsymbol{\sigma} \mathbf{n} d \gamma
$$

To obtain a distributed Lagrange multiplier/fictitious domain formulation for the above problem (1)-(10), we proceed as in [12, 13], namely: (i) we first obtain a variational formulation of the problem (1)-(10), (ii) we then fill the region occupied by the rigid body by the surrounding fluid (i.e., embed $\Omega \backslash \overline{B(t)}$ in $\Omega$ ) with the constraint, that the fluid inside the rigid body region should have a rigid body motion, and then (iii) we relax the rigid body motion constraint by using a distributed Lagrange multiplier, obtaining thus a fictitious domain formulation over the entire region $\Omega$ as follows:

For a.e. $t \in(0, T)$, find $\mathbf{u}(t) \in \mathbf{V}_{\mathbf{g}_{0}}, p(t) \in L_{0}^{2}(\Omega), \mathbf{V}(t) \in \mathbb{R}^{2}, \mathbf{G}(t) \in \mathbb{R}^{2}, \omega(t) \in \mathbb{R}$, $\theta(t) \in \mathbb{R}, \boldsymbol{\lambda}(t) \in \Lambda(t)$ such that

$$
\left\{\begin{array}{l}
-\int_{\Omega} p \mathbf{\nabla} \cdot \mathbf{v} d \mathbf{x}+2 \mu_{f} \int_{\Omega} \mathbf{D}(\mathbf{u}): \mathbf{D}(\mathbf{v}) d \mathbf{x} \\
-<\boldsymbol{\lambda}, \mathbf{v}-\mathbf{Y}-\xi \overrightarrow{\mathbf{G x}}{ }^{\perp}>_{\Lambda(t)}+M_{p} \frac{d \mathbf{V}}{d t} \cdot \mathbf{Y}+I_{p} \frac{d \omega}{d t} \xi \\
=\left(1-\frac{\rho_{f}}{\rho_{s}}\right) M_{p} \mathbf{g} \cdot \mathbf{Y}+\rho_{f} \int_{\Omega} \mathbf{g} \cdot \mathbf{v} d \mathbf{x}, \\
\quad \forall \mathbf{v} \in \mathbf{V}_{0}, \quad \forall \mathbf{Y} \in \mathbb{R}^{2}, \quad \forall \xi \in \mathbb{R},
\end{array}\right.
$$




$$
\begin{aligned}
& \int_{\Omega} q \boldsymbol{\nabla} \cdot \mathbf{u}(t) d \mathbf{x}=0, \forall q \in L^{2}(\Omega), \\
& \frac{d \mathbf{G}}{d t}=\mathbf{V}, \\
& \frac{d \theta}{d t}=\omega, \\
& <\boldsymbol{\mu}, \mathbf{u}(t)-\mathbf{V}(t)-\omega(t) \overrightarrow{\mathbf{G x}}^{\perp}>_{\Lambda(t)}=0, \forall \boldsymbol{\mu} \in \Lambda(t), \\
& \mathbf{V}(0)=\mathbf{V}_{0}, \omega(0)=\omega_{0}, \mathbf{G}(0)=\mathbf{G}_{0}, \theta(0)=\theta_{0}
\end{aligned}
$$

where the function spaces in problem (11)-(16) are defined as

$$
\begin{aligned}
& \mathbf{V}_{\mathbf{g}_{0}}=\left\{\mathbf{v} \mid \mathbf{v} \in\left(H^{1}(\Omega)\right)^{2}, \mathbf{v}=\mathbf{g} \text { on } \Gamma, \mathbf{v} \text { is periodic in the } x_{1}\right. \\
& \text { direction with period } L\} \text {, } \\
& \mathbf{V}_{0}=\left\{\mathbf{v} \mid \mathbf{v} \in\left(H^{1}(\Omega)\right)^{2}, \mathbf{v}=\mathbf{0} \text { on } \Gamma, \mathbf{v} \text { is periodic in the } x_{1}\right. \\
& \text { direction with period } L\} \text {, } \\
& L_{0}^{2}(\Omega)=\left\{q \mid q \in L^{2}(\Omega), \int_{\Omega} q d \mathbf{x}=0\right\}, \\
& \Lambda(t)=\left(H^{1}(B(t))\right)^{2},
\end{aligned}
$$

and for any $\boldsymbol{\mu} \in H^{1}(B(t))^{2}$ and any $\mathbf{v} \in \mathbf{V}_{0}$, the inner product $<\cdot, \cdot>_{\Lambda(t)}$ in (11) and (15) is defined by

$$
<\boldsymbol{\mu}, \mathbf{v}>_{\Lambda(t)}=\int_{B(t)}\left(\boldsymbol{\mu} \cdot \mathbf{v}+\frac{1}{d^{2}} \boldsymbol{\nabla} \boldsymbol{\mu}: \nabla \mathbf{v}\right) d \mathbf{x}
$$

where $d$ is a scaling constant and, typically, the diameter of the particle is used as $d$.

Remark 1. In relation (11) we can replace $2 \int_{\Omega} \mathbf{D}(\mathbf{u}): \mathbf{D}(\mathbf{v}) d \mathbf{x}$ by $\int_{\Omega} \nabla \mathbf{u}: \nabla \mathbf{v} d \mathbf{x}$. Also the gravity $\mathbf{g}$ in (11) can be absorbed into the pressure term. For the cases of Poiseuille flow, a body force $\mathbf{f}_{p}$, which is the pressure gradient pointing in the $x_{1}$ direction, is added to the equation (11). For the cases of compound vesicle (a neutrally buoyant particle enclosed by a membrane), we add the term $\mathbf{f}_{B}$ for the force acting on the fluid/cell interface to the equation (11) (see the following subsection).

Remark 2. The system (11)-(16) is quite different from those considered in, e.g., $[15,20]$ for the cases of neutrally buoyant particles in incompressible viscous flow modeled by Navier-Stokes equations. For the particle-flow interaction under creeping flow in this article, we do not need to add any extra constraint on the Lagrange multiplier as in $[15,20]$.

\subsection{An elastic spring model}

An elastic spring model similar to the one used in [23] is considered in this article to describe the deformable behavior and elasticity of vesicle. Based on this model, 


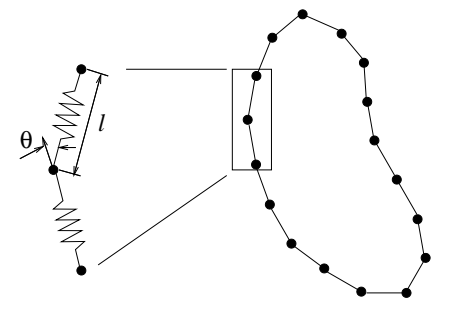

Figure 2: The elastic spring model of the membrane.

the membrane can be viewed as membrane particles connecting with the neighboring membrane particles by springs, as shown in Figure 2. Energy stores in the spring due to the change of the length $l$ of the spring with respect to its reference length $l_{0}$ and the change in angle $\theta$ between two neighboring springs. The total energy per unit thickness of the membrane, $E=E_{l}+E_{b}$, is the sum of the one for stretch and compression and the one for the bending which, typically, are

$$
E_{l}=\frac{k_{l}}{2} \sum_{i=1}^{N}\left(\frac{l_{i}-l_{0}}{l_{0}}\right)^{2}, \quad E_{b}=\frac{k_{b}}{2} \sum_{i=1}^{N} \tan ^{2}\left(\frac{\theta_{i}}{2}\right) .
$$

In equation (17), $N$ is the total number of spring elements, and $k_{l}$ and $k_{b}$ are the spring constants for changes in length and bending angle, respectively.

To obtain a specified initial shape for the fluid-vesicle interaction, the vesicle is assumed to be, initially, a circle of radius $R_{0}=2.8 \mu \mathrm{m}$ and then it is discretized into $N=76$ membrane particles so that 76 springs of equal lengths are formed by connecting the neighboring particles. The shape change is stimulated by reducing the total area enclosed by the membrane through a penalty function

$$
\Gamma_{s}=\frac{k_{s}}{2}\left(\frac{s-s_{e}}{s_{e}}\right)^{2}
$$

where $s$ and $s_{e}$ are the time dependent area enclosed by the membrane and the targeted area enclosed by the membrane, respectively, the total energy per unit thickness being modified as $E+\Gamma_{s}$. Based on the principle of virtual work the force per unit thickness acting on the $i$ th membrane particle now is

$$
\mathbf{F}_{i}=-\frac{\partial\left(E+\Gamma_{s}\right)}{\partial \mathbf{r}_{i}}
$$

where $\mathbf{r}_{i}$ is the position of the $i$ th membrane particle. When the area is reduced, each membrane particle moves on the basis of the following equation of motion:

$$
m \frac{d^{2} \mathbf{r}_{i}}{d t^{2}}+\gamma \frac{d \mathbf{r}_{i}}{d t}=\mathbf{F}_{i}
$$


Here $m$ and $\gamma$ represent the membrane particle mass and the membrane viscosity. The position $\mathbf{r}_{i}$ of the $i$ th membrane particle is solved by discretizing equation (20) via a second order finite difference method. The total energy stored in the membrane decreases as the time elapses. The final shape of the vesicle is obtained as the total energy is minimized and such shape is at a stress-free state. In equations (17) and (18), $E_{l}, E_{b}$, and $\Gamma_{s}$ represent energies [ $\mathrm{N} \mathrm{m}$ ] per unit thickness of $1 \mathrm{~m}$, and thus the units of $k_{l}, k_{b}$, and $k_{s}$ are Newton [N]. The value of the swelling ratio of a vesicle is $s^{*}=s_{e} /\left(\pi R_{0}^{2}\right)$. The values of the parameters used for modeling the vesicles are as follows: The spring constant is $k_{l}=5 \times 10^{-8} \mathrm{~N}$, the penalty coefficient is $k_{s}=10^{-5}$ $\mathrm{N}$, and the bending constant is $k_{b}=5 \times 10^{-10} \mathrm{~N}$. Using the above chosen parameter values, the area of the initial shape has less than $0.001 \%$ difference from the given equilibrium area $s_{e}$, and the length of the perimeter of the initial shape has less than $0.005 \%$ difference from the circumference of the initial circle.

Remark 3. When considering the cases of compound vesicle, a repulsion force $\mathbf{F}^{r}$ and associated torque $T^{r}$ are added to equations (7) and (8), respectively, and then to (11) to prevent the particle-cell penetration. Such repulsive force is given by a Morse potential (e.g., see [24])

$$
\phi(s)=k_{r}\left(1-e^{-\left(s-s_{0}\right)}\right)^{2}
$$

where $s$ is the shortest distance from a membrane node to the surface of the solid particle and $s_{0}$ is the range of the repulsive force (when the distance $s$ is greater than $s_{0}$, there is no repulsive force). The parameter $k_{r}$ is a constant for the strength of the potential. In our simulations, we have used $k_{r}=8 \times 10^{-8} \mathrm{~N}$ and $s_{0}=2 h$ where $h$ is the mesh size. We first compute $\mathbf{f}_{i}^{r}=-\frac{\partial \phi(s)}{\partial s}$ for each membrane node $\mathbf{r}_{i}$ of iRBC membrane and associated torque $\mathbf{f}_{i}^{r} \cdot \overrightarrow{\mathbf{G x}_{i}}{ }^{\perp}$ where $\mathbf{x}_{i}$ is the closest point on the particle surface to the membrane node $\mathbf{r}_{i}$ and then sum up all of them to obtain $\mathbf{F}^{r}$ and the associated torque $T^{r}$.

\section{$2.3 \quad$ Numerical methods}

For the space discretization, we have chosen $P_{1}-i$ so- $P_{2}$ and $P_{1}$ finite element spaces for the velocity field and pressure, respectively, (like in Bristeau et al. [25] and Glowinski $[26]$ ), that is

$$
\begin{aligned}
& \mathbf{W}_{h}=\left\{\mathbf{v}_{h}\left|\mathbf{v}_{h} \in\left(C^{0}(\bar{\Omega})\right)^{2}, \mathbf{v}_{h}\right|_{T} \in\left(P_{1}\right)^{2}, \forall T \in \mathcal{T}_{h},\right. \\
&\left.\mathbf{v} \text { is periodic in the } x_{1} \text { direction with period } L\right\}, \\
& \mathbf{W}_{0 h}=\left\{\mathbf{v}_{h} \mid \mathbf{v}_{h} \in \mathbf{W}_{h}, \mathbf{v}_{h}=\mathbf{0} \text { on } \Gamma\right\}, \\
& L_{h}^{2}=\left\{q_{h}\left|q_{h} \in C^{0}(\bar{\Omega}), q_{h}\right|_{T} \in P_{1}, \forall T \in \mathcal{T}_{2 h}\right\}, \\
& L_{0 h}^{2}=\left\{q_{h} \mid q_{h} \in L_{h}^{2}, \int_{\Omega} q_{h} d \mathbf{x}=0\right\}
\end{aligned}
$$


where $h$ is space mesh size, $\mathcal{T}_{h}$ is a structured triangulation of $\Omega, \mathcal{T}_{2 h}$ is twice coarser than $\mathcal{T}_{h}$, and $P_{1}$ is the space of the polynomials in two variables of degree $\leq 1$.

A finite dimensional space approximating $\Lambda(t)$ is defined as follows: let $\left\{\mathbf{x}_{i}\right\}_{i=1}^{N}$ be a set of points covering $\overline{B(t)}$; the discrete multiplier space $\Lambda_{h}(t)$ is defined by

$$
\Lambda_{h}(t)=\left\{\boldsymbol{\mu}_{h} \mid \boldsymbol{\mu}_{h}=\sum_{i=1}^{N} \boldsymbol{\mu}_{i} \delta\left(\mathbf{x}-\mathbf{x}_{i}\right), \boldsymbol{\mu}_{i} \in \mathbb{R}^{2}, \forall i=1, \ldots, N\right\},
$$

where $\delta(\cdot)$ is the Dirac measure at $\mathbf{x}=\mathbf{0}$. Then, we define an inner product over $\Lambda_{h}(t)$ by

$$
<\boldsymbol{\mu}_{h}, \mathbf{v}_{h}>_{\Lambda_{h}(t)}=\sum_{i=1}^{N} \boldsymbol{\mu}_{i} \cdot \mathbf{v}_{h}\left(\mathbf{x}_{i}\right), \forall \boldsymbol{\mu}_{h} \in \Lambda_{h}(t), \mathbf{v}_{h} \in \mathbf{W}_{0 h}
$$

The immersed boundary method developed by Peskin, e.g, [27, 28, 29], is employed in this study because of its distinguished features in dealing with the problem of fluid flow interacting with a flexible fluid/structure interface. Over the years, it has demonstrated its CFD capability including blood flow simulations. Based on the method, the boundary of the deformable structure is discretized spatially into a set of boundary nodes. The force located at the immersed boundary node $\mathbf{r}_{i}=\left(r_{i, 1}, r_{i, 2}\right)$ affects the nearby fluid mesh nodes $\mathbf{x}=\left(x_{1}, x_{2}\right)$ through a $2 \mathrm{D}$ discrete $\delta$-function $D_{h}\left(\mathbf{x}-\mathbf{r}_{i}\right)$ :

$$
\mathbf{f}_{B}(\mathbf{x})=\sum \mathbf{F}_{i} D_{h}\left(\mathbf{x}-\mathbf{r}_{i}\right) \text { for }\left|\mathbf{x}-\mathbf{r}_{i}\right| \leq 2 h,
$$

where $h$ is the uniform finite element mesh size and

$$
D_{h}\left(\mathbf{x}-\mathbf{r}_{i}\right)=\delta_{h}\left(x_{1}-r_{i, 1}\right) \delta_{h}\left(x_{2}-r_{i, 2}\right),
$$

the 1D discrete $\delta$-functions being defined by

$$
\delta_{h}(z)= \begin{cases}\frac{1}{8 h}\left(3-2|z| / h+\sqrt{1+4|z| / h-4(|z| / h)^{2}}\right), & |z| \leq h \\ \frac{1}{8 h}\left(5-2|z| / h-\sqrt{-7+12|z| / h-4(|z| / h)^{2}}\right), & h \leq|z| \leq 2 h \\ 0, & \text { otherwise }\end{cases}
$$

The velocity of the immersed boundary node $\mathbf{r}_{i}$ is also affected by the surrounding fluid and therefore is enforced by summing the velocities at the nearby fluid mesh nodes $\mathbf{x}$ weighted by the same discrete $\delta$-function:

$$
\mathbf{U}\left(\mathbf{r}_{i}\right)=\sum h^{2} \mathbf{u}(\mathbf{x}) D_{h}\left(\mathbf{x}-\mathbf{r}_{i}\right) \text { for }\left|\mathbf{x}-\mathbf{r}_{i}\right| \leq 2 h .
$$

After each time step, the position of the immersed boundary node is updated by

$$
\mathbf{r}_{i}^{n+1}=\mathbf{r}_{i}^{n}+\Delta t \mathbf{U}\left(\mathbf{r}_{i}^{n}\right)
$$


Applying the above finite dimensional spaces (after dropping $h$ 's) for space discretization and Lie's scheme [30] in time advancing (see, e.g., [17, 19, 20] for similar approaches) to the problem (11)-(16) with an immersed boundary method and the backward Euler method in time for the last subproblem, we have the following sequence of subproblems:

$\mathbf{u}^{0}=\mathbf{u}_{0}, \mathbf{G}^{0}=\mathbf{G}_{0}, \mathbf{V}^{0}=\mathbf{V}_{0}, \omega^{0}=\omega_{0}, \theta^{0}=\theta_{0}$ are given; for $n \geq 0, \mathbf{u}^{n}, \mathbf{G}^{n}$, $\mathbf{V}^{n}, \omega^{n}$ and $\theta^{n}$ being known, we compute the approximate solution via the following fractional steps:

1. Update the position of the membrane by (30) and (31). Then compute the force $\mathbf{f}_{B}^{n+1}$ on the fluid/cell interface by (19) and (27).

2. We predict the motion of the particle via

$$
\begin{aligned}
& \frac{d \mathbf{G}}{d t}=\mathbf{V}(t), \\
& \frac{d \theta}{d t}=\omega(t), \\
& M_{p} \frac{d \mathbf{V}}{d t}=\mathbf{F}^{r}(t) \\
& I_{p} \frac{d \omega}{d t}=T^{r}(t), \\
& \mathbf{V}\left(t^{n}\right)=\mathbf{V}^{n}, \omega\left(t^{n}\right)=\omega^{n}, \mathbf{G}\left(t^{n}\right)=\mathbf{G}^{n}, \theta\left(t^{n}\right)=\theta^{n}
\end{aligned}
$$

for $t^{n}<t<t^{n+1}$. Then set $\mathbf{V}^{n+\frac{1}{2}}=\mathbf{V}\left(t^{n+1}\right), \omega^{n+\frac{1}{2}}=\omega\left(t^{n+1}\right), \mathbf{G}^{n+1}=\mathbf{G}\left(t^{n+1}\right)$ and $\theta^{n+1}=\theta\left(t^{n+1}\right)$.

3. With the center $\mathbf{G}^{n+1}$ and inclination angle $\theta^{n+1}$ obtained in the above step, the region of $B^{n+1}$ occupied by the particle is determined. Then we enforce the rigid body motion in $B^{n+1}$ and solve $\mathbf{u}^{n+1}$ and $p^{n+1}$ at the same time as follows: Find $\mathbf{u}^{n+1} \in \mathbf{W}_{h}, \mathbf{u}^{n+1}=\mathbf{g}_{0}$ on $\Gamma, p^{n+1} \in L_{0 h}^{2}, \boldsymbol{\lambda}^{n+1} \in \Lambda_{h}^{n+1}, \mathbf{V}^{n+1} \in \mathbb{R}^{2}$, $\omega^{n+1} \in \mathbb{R}$ so that

$$
\begin{aligned}
& \left\{\begin{array}{l}
-\int_{\Omega} p^{n+1} \nabla \cdot \mathbf{v} d \mathbf{x}+\mu_{f} \int_{\Omega} \boldsymbol{\nabla} \mathbf{u}^{n+1}: \nabla \mathbf{v} d \mathbf{x} \\
+M_{p} \frac{\mathbf{V}^{n+1}-\mathbf{V}^{n+\frac{1}{2}}}{\triangle t} \cdot \mathbf{Y}+I_{p} \frac{\omega^{n+1}-\omega^{n+\frac{1}{2}}}{\triangle t} \xi \\
=\left(1-\frac{\rho_{f}}{\rho_{s}}\right) M_{p} \mathbf{g} \cdot \mathbf{Y}+\int_{\Omega} \mathbf{f}^{n+1} \cdot \mathbf{v} d \mathbf{x}+<\boldsymbol{\lambda}^{n+1}, \mathbf{v}-\mathbf{Y}-\xi \overrightarrow{\mathbf{G}^{n+1} \mathbf{x}}>_{\Lambda_{h}^{n+1}} \\
\forall \mathbf{v} \in \mathbf{W}_{0 h}, \mathbf{Y} \in \mathbb{R}^{2}, \xi \in \mathbb{R}
\end{array}\right. \\
& \int_{\Omega} q \boldsymbol{\nabla} \cdot \mathbf{u}^{n+1} d \mathbf{x}=0, \forall q \in L_{h}^{2}, \\
& <\boldsymbol{\mu}, \mathbf{u}^{n+1}-\mathbf{V}^{n+1}-\omega^{n+1} \overrightarrow{\mathbf{G}^{n+1} \mathbf{x}}>_{\Lambda_{h}^{n+1}}=0, \forall \boldsymbol{\mu} \in \Lambda_{h}^{n+1}
\end{aligned}
$$


In equations (37)-(39), we have $\Lambda_{h}^{n+1}=\Lambda_{h}\left(t^{n+1}\right)$ and $\mathbf{f}^{n+1}=\mathbf{f}_{B}^{n+1}$ (or $\mathbf{f}^{n+1}=\mathbf{f}_{B}^{n+1}+\mathbf{f}_{p}$ when considering Poiseuille flow).

Remark 4. When simulating particle motion in fluid flow under creeping flow condition, we only use steps 2 and 3 in the above algorithm without having to consider the repulsion force between the particle and membrane and the force $\mathbf{f}_{B}^{n+1}$ on the fluid/cell interface.

\subsection{On the solution of the subproblems}

System (32)-(36) is just a system of ordinary differential equations. These equations are solved by the forward Euler method with the time step $\triangle t$ to predict the translation velocity of the center of mass, the angular velocity of the particle, the position of the center of mass and the inclination angle of the particle of a long body shape.

In system (37)-(39), there are two multipliers, $p$ and $\boldsymbol{\lambda}$. We have solved this system via an Uzawa-conjugate gradient method driven by both multipliers (a one shot method, similar to those discussed in, e.g., [31, 32]). The general problem is as follows:

Find $\mathbf{u} \in \mathbf{W}_{h}, \mathbf{u}=\mathbf{g}_{0}$ on $\Gamma, p \in L_{0 h}^{2}, \boldsymbol{\lambda} \in \Lambda_{h}, \mathbf{V} \in \mathbb{R}^{2}, \omega \in \mathbb{R}$ so that

$$
\begin{aligned}
& \left\{\begin{array}{l}
-\int_{\Omega} p \boldsymbol{\nabla} \cdot \mathbf{v} d \mathbf{x}+\mu_{f} \int_{\Omega} \nabla \mathbf{u}: \nabla \mathbf{v} d \mathbf{x}+M_{p} \frac{\mathbf{V}-\mathbf{V}_{0}}{\Delta t} \cdot \mathbf{Y}+I_{p} \frac{\omega-\omega_{0}}{\Delta t} \xi \\
\quad=\left(1-\frac{\rho_{f}}{\rho_{s}}\right) M_{p} \mathbf{g} \cdot \mathbf{Y}+\int_{\Omega} \mathbf{f} \cdot \mathbf{v} d \mathbf{x}+<\boldsymbol{\lambda}, \mathbf{v}-\mathbf{Y}-\xi \overrightarrow{\mathbf{G x}}{ }^{\perp}>_{\Lambda_{h}}, \\
\forall \mathbf{v} \in \mathbf{W}_{0 h}, \mathbf{Y} \in \mathbb{R}^{2}, \xi \in \mathbb{R},
\end{array}\right. \\
& \int_{\Omega} q \nabla \cdot \mathbf{u} d \mathbf{x}=0, \forall q \in L_{h}^{2}, \\
& <\boldsymbol{\mu}, \mathbf{u}-\mathbf{V}-\omega \overrightarrow{\mathbf{G X}}^{\perp}>_{\Lambda_{h}}=0, \forall \boldsymbol{\mu} \in \Lambda_{h} .
\end{aligned}
$$

We apply the following Uzawa-conjugate gradient algorithm operating in the space $L_{0 h}^{2} \times \Lambda_{h}$ to solve the above problem:

$$
p^{0} \in L_{0 h}^{2} \text { and } \boldsymbol{\lambda}^{0} \in \Lambda_{h} \text { are given; }
$$

solve

$$
\begin{aligned}
& \left\{\begin{array}{l}
\mu_{f} \int_{\Omega} \nabla \mathbf{u}^{0}: \nabla \mathbf{v} d \mathbf{x}=\int_{\Omega} p^{0} \nabla \cdot \mathbf{v} d \mathbf{x}+\int_{\Omega} \mathbf{f} \cdot \mathbf{v} d \mathbf{x}+<\boldsymbol{\lambda}^{0}, \mathbf{v}>_{\Lambda_{h}}, \\
\forall \mathbf{v} \in \mathbf{W}_{0 h} ; \mathbf{u}^{0} \in \mathbf{W}_{h}, \mathbf{u}=\mathbf{g}_{0 h} \text { on } \Gamma,
\end{array}\right. \\
& M_{p} \frac{\mathbf{V}^{0}-\mathbf{V}_{0}}{\triangle t} \cdot \mathbf{Y}=\left(1-\frac{\rho_{f}}{\rho_{s}}\right) M_{p} \mathbf{g} \cdot \mathbf{Y}-<\boldsymbol{\lambda}^{0}, \mathbf{Y}>_{\Lambda_{h}}, \forall \mathbf{Y} \in \mathbb{R}^{2}, \\
& I_{p} \frac{\omega^{0}-\omega_{0}}{\triangle t}=-<\boldsymbol{\lambda}^{0}, \overrightarrow{\mathbf{G} \mathbf{x}}{ }^{\perp}>_{\Lambda_{h}},
\end{aligned}
$$


and then compute

$$
g_{1}^{0}=\nabla \cdot \mathbf{u}^{0}
$$

next solve

$$
\left\{\begin{array}{l}
\mathbf{g}_{2}^{0} \in \Lambda_{h}, \\
<\boldsymbol{\mu}, \mathbf{g}_{2}^{0}>_{\Lambda_{h}}=<\boldsymbol{\mu}, \mathbf{u}^{0}-\mathbf{V}^{0}-\omega^{0} \overrightarrow{\mathbf{G x}^{\perp}}>_{\Lambda_{h}}, \forall \boldsymbol{\mu} \in \Lambda_{h},
\end{array}\right.
$$

and set

$$
w_{1}^{0}=g_{1}^{0}, \text { and } \mathbf{w}_{2}^{0}=\mathbf{g}_{2}^{0} .
$$

Then for $k \geq 0$, assuming that $p^{k}, \boldsymbol{\lambda}^{k}, \mathbf{u}^{k}, \mathbf{V}^{k}, \omega^{k}, g_{1}^{k}, \mathbf{g}_{2}^{k}, w_{1}^{k}$ and $\mathbf{w}_{2}^{k}$ are known, compute $p^{k+1}, \boldsymbol{\lambda}^{k+1}, \mathbf{u}^{k+1}, \mathbf{V}^{k+1}, \omega^{k+1}, g_{1}^{k+1}, \mathbf{g}_{2}^{k+1}, w_{1}^{k+1} \mathbf{w}_{2}^{k+1}$ as follows:

solve:

$$
\begin{aligned}
& \left\{\begin{array}{l}
\mu_{f} \int_{\Omega} \nabla \overline{\mathbf{u}}^{k}: \nabla \mathbf{v} d \mathbf{x}=\int_{\Omega} w_{1}^{k} \nabla \cdot \mathbf{v} d \mathbf{x}+<\mathbf{w}_{2}^{k}, \mathbf{v}>_{\Lambda_{h}}, \\
\forall \mathbf{v} \in \mathbf{W}_{0 h} ; \overline{\mathbf{u}}^{k} \in \mathbf{W}_{0 h},
\end{array}\right. \\
& M_{p} \frac{\overline{\mathbf{V}}^{k}}{\triangle t} \cdot \mathbf{Y}=-<\mathbf{w}_{2}^{k}, \mathbf{Y}>_{\Lambda_{h}}, \forall \mathbf{Y} \in \mathbb{R}^{2}, \\
& I_{p} \frac{\bar{\omega}^{k}}{\Delta t}=-<\mathbf{w}_{2}^{k}, \quad \overrightarrow{\mathbf{G X}}^{\perp}>_{\Lambda_{h}},
\end{aligned}
$$

and then compute

$$
\bar{g}_{1}^{k}=\nabla \cdot \overline{\mathbf{u}}^{k}
$$

next solve

$$
\left\{\begin{array}{l}
\overline{\mathbf{g}}_{2}^{k} \in \Lambda_{h}, \\
<\boldsymbol{\mu}, \overline{\mathbf{g}}_{2}^{k}>_{\Lambda_{h}}=<\boldsymbol{\mu}, \overline{\mathbf{u}}^{k}-\overline{\mathbf{V}}^{k}-\bar{\omega}^{k} \overrightarrow{\mathbf{G x}}{ }^{\perp}>_{\Lambda_{h}}, \forall \boldsymbol{\mu} \in \Lambda_{h},
\end{array}\right.
$$

and compute

$$
\rho_{k}=\frac{\int_{\Omega}\left|g_{1}^{k}\right|^{2} d \mathbf{x}+<\mathbf{g}_{2}^{k}, \mathbf{g}_{2}^{k}>_{\Lambda_{h}}}{\int_{\Omega} \bar{g}_{1}^{k} w_{1}^{k} d \mathbf{x}+<\overline{\mathbf{g}}_{2}^{k}, \mathbf{w}_{2}^{k}>_{\Lambda_{h}}}
$$

and

$$
\begin{aligned}
& p^{k+1}=p^{k}-\rho_{k} w_{1}^{k}, \\
& \boldsymbol{\lambda}^{k+1}=\boldsymbol{\lambda}^{k}-\rho_{k} \mathbf{w}_{2}^{k}, \\
& \mathbf{u}^{k+1}=\mathbf{u}^{k}-\rho_{k} \overline{\mathbf{u}}^{k}, \\
& \mathbf{V}^{k+1}=\mathbf{V}^{k}-\rho_{k} \overline{\mathbf{V}}^{k}, \\
& \omega^{k+1}=\omega^{k}-\rho_{k} \bar{\omega}^{k}, \\
& g_{1}^{k+1}=g_{1}^{k}-\rho_{k} \bar{g}_{1}^{k}, \\
& \mathbf{g}_{2}^{k+1}=\mathbf{g}_{2}^{k}-\rho_{k} \mathbf{g}_{2}^{k} .
\end{aligned}
$$


If

$$
\frac{\int_{\Omega}\left|g_{1}^{k+1}\right|^{2} d \mathbf{x}+<\mathbf{g}_{2}^{k+1}, \mathbf{g}_{2}^{k+1}>_{\Lambda_{h}}}{\int_{\Omega}\left|g_{1}^{0}\right|^{2} d \mathbf{x}+<\mathbf{g}_{2}^{0}, \mathbf{g}_{2}^{0}>_{\Lambda_{h}}} \leq t o l
$$

take $p=p^{k+1}, \boldsymbol{\lambda}=\boldsymbol{\lambda}^{k+1}, \mathbf{u}=\mathbf{u}^{k+1}, \mathbf{V}=\mathbf{V}^{k+1}, \omega=\omega^{k+1} ;$ else, compute

$$
\gamma_{k}=\frac{\int_{\Omega}\left|g_{1}^{k+1}\right|^{2} d \mathbf{x}+<\mathbf{g}_{2}^{k+1}, \mathbf{g}_{2}^{k+1}>_{\Lambda_{h}}}{\int_{\Omega}\left|g_{1}^{k}\right|^{2} d \mathbf{x}+<\mathbf{g}_{2}^{k}, \mathbf{g}_{2}^{k}>_{\Lambda_{h}}}
$$

and set

$$
\begin{aligned}
& w_{1}^{k+1}=g_{1}^{k+1}+\gamma_{k} w_{1}^{k}, \\
& \mathbf{w}_{2}^{k+1}=\mathbf{g}_{2}^{k+1}+\gamma_{k} \mathbf{w}_{2}^{k} .
\end{aligned}
$$

Do $k=k+1$ and go back to (49).

\section{$3 \quad$ Numerical results}

\subsection{Particle motion in simple shear flow}

First we have considered the cases of a neutrally buoyant particle of circular shape located at the middle between two walls initially. The circular particle radius $a$ varies from 0.1 to 0.25 . The densities of the fluid and that of the particle are $\rho_{f}=\rho_{s}=1$ and the viscosity is $\mu_{f}=1$. The confined ratio is defined as $\kappa=2 a / H$ where $H$ is the distance between two walls. The computational domain is $\Omega=[0,2] \times[0,2]$ (i.e., $L=2$ and $H=2$ ). The shear rate is fixed at $G_{s}=1$ so the velocity of the top wall (resp., bottom wall) is $U / 2=G_{s} H / 2=1$ (resp., $-U / 2=-1$ ). The initial velocity field is a simple shear flow associated to the given shear rate without particle. The mass center of the circular particle is located at $(1,1)$ initially. The space mesh size is $h=1 / 128$ and the time step is $\triangle=0.001$. Under creeping flow condition, the rotating velocity of the circular particle is $-G_{s} / 2$ in an unbounded shear flow according to the Jeffery's solution [33]. In Figure 3, a snapshot of the velocity filed of the case $\kappa=0.25(a=0.25)$ is shown and the plot of the rotation speed versus the confined ratio is also presented. The confined ratio does have its effect on the rotation speed as shown in Figure 3. For the cases of the cylinder radii $a=0.1,0.125$ and 0.15 , the relative errors on the rotation speed are less than or equal to $1 \%$. These computed angular speeds are in a good agreement with the Jeffery's solution.

Next we have considered the motion of an elliptic shape particle in shear flow. The particle mass center is located at the middle between two walls initially. The semi-major and semi-minor axes are 0.1 and 0.05 . The other parameters are same as those in the first cases except that two space mesh sizes of 1/128 and 1/192 are used and the initial inclination angle is $\pi / 4$. The computed rotating velocities have been 

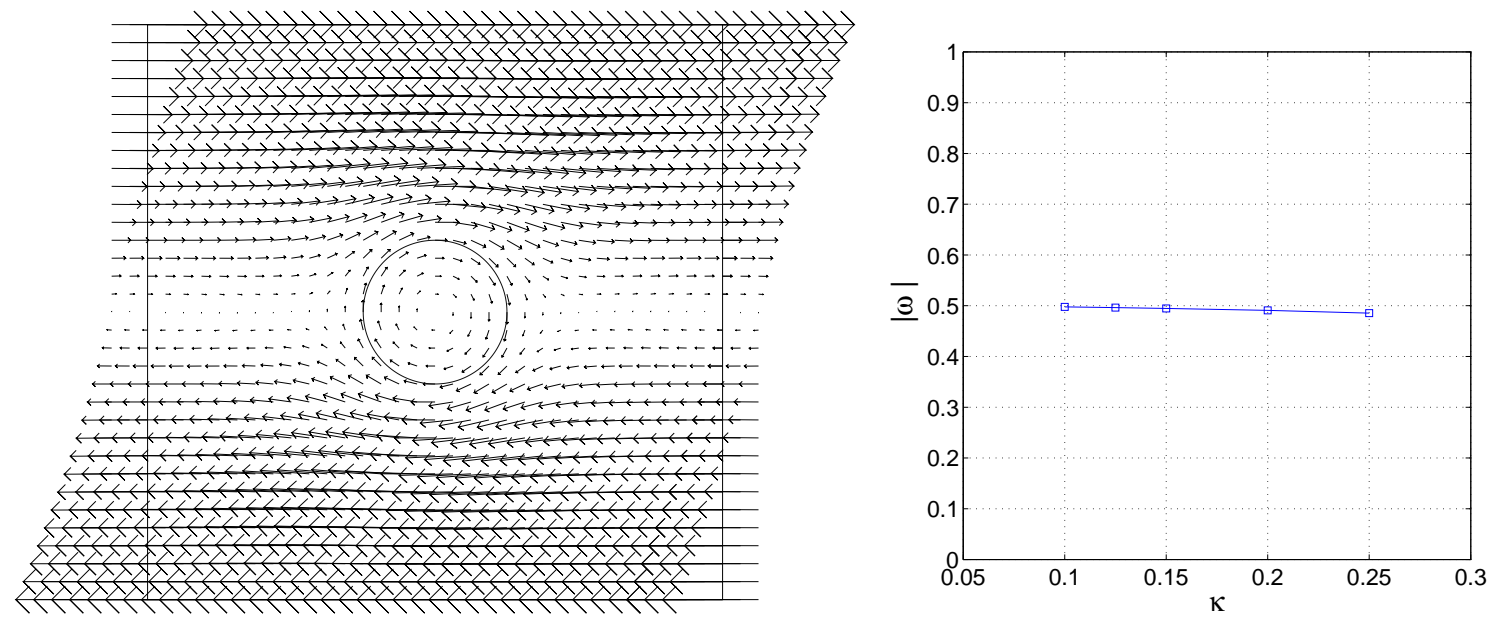

Figure 3: The velocity field next to the neutrally buoyant disk for the case $\kappa=0.25$ (left) and the rotating speed versus the confined ratio (right).
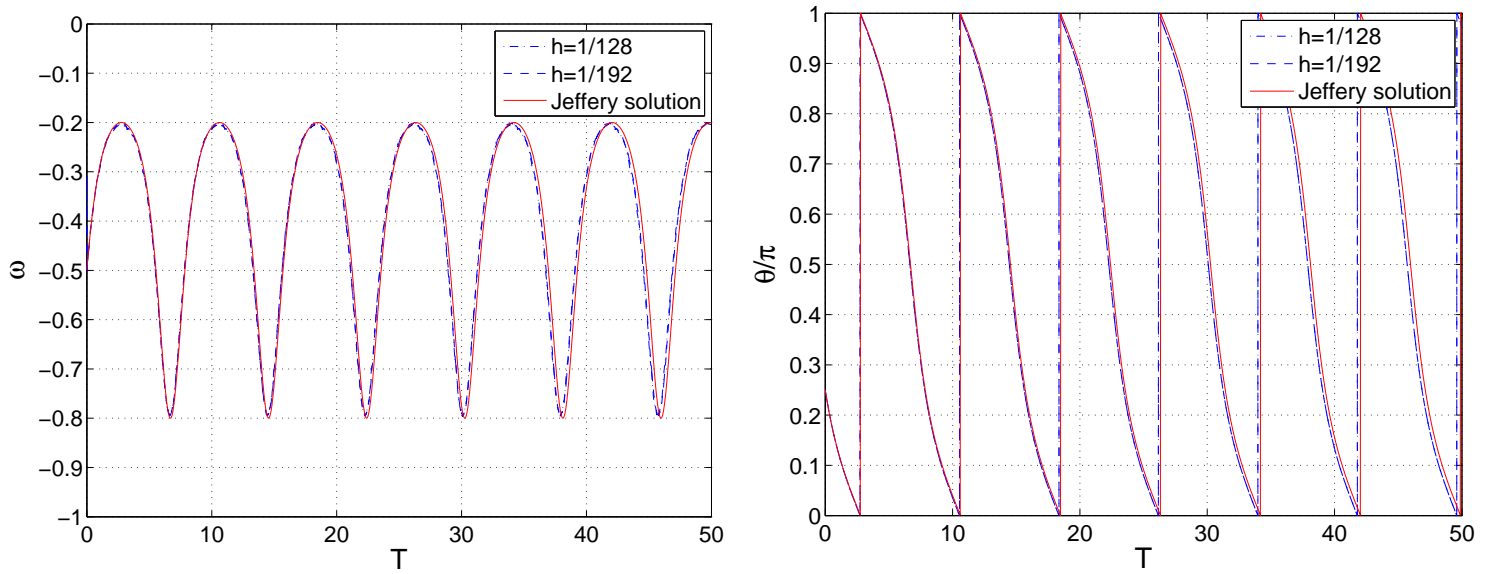

Figure 4: Histories of the rotating velocity (left) and the inclination angle (right) of an elliptic shape particle in simple shear flow obtained with mesh sizes $h=1 / 128$ and $1 / 192$.

compared with the Jeffery's solution in Figure 4. A snapshot of the velocity field is shown in Figure 5. The maximum and minimum rotating speeds of the Jeffery's solution in magnitude are 0.8 and 0.2 , while ours are 0.795 and 0.201 . The histories of the rotating velocity and inclination angle are shown in Figure 4 (due to the axisymmetric shape of the elliptical particle, the range of the inclination angle in the plot is from zero to $\pi$ ). Both agree well with the Jeffery's solution. 


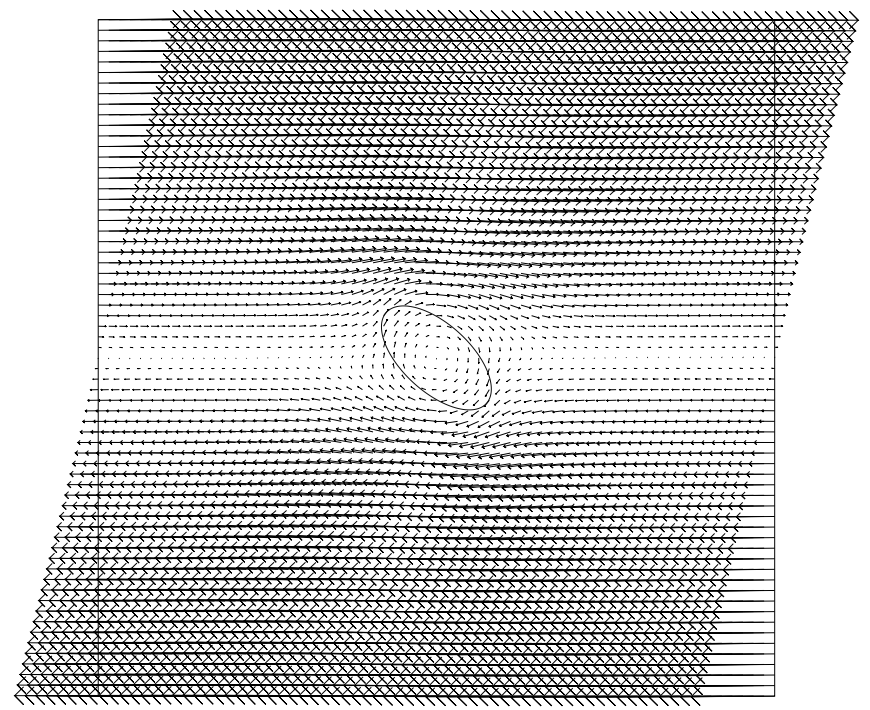

Figure 5: A snapshot of the velocity field next to the elliptic shape particle.
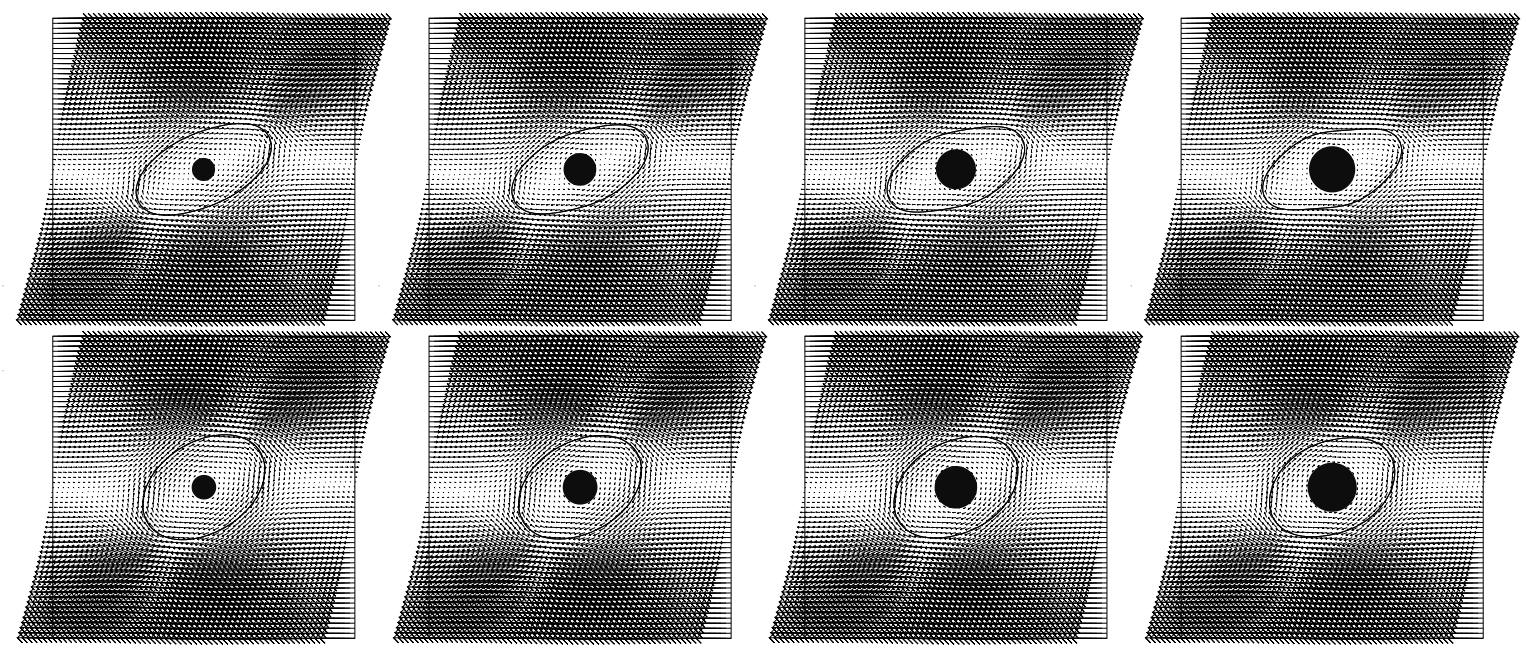

Figure 6: Snapshots of velocity field next to a compound vesicle of swelling ratio $s^{*}=0.84$ (top) and 0.95 (bottom) with filling fraction $\phi=0.05,0.1,0.15$, and 0.2 (from left to right) at the shear rate $G_{s}=250 / \mathrm{s}$.

\subsection{Motion of a compound vesicle in fluid flow}

First we have considered the cases of a compound vesicle with a circular shape particle in shear flow. The computational domain is $\Omega=20 \times 20 \mu \mathrm{m}^{2}$. Two initial shapes for the vesicle are obtained by the procedure discussed in Section 2.2 with the swelling ratio $s^{*}=0.84$ and 0.95 , respectively. With the parameters of the membrane as in 

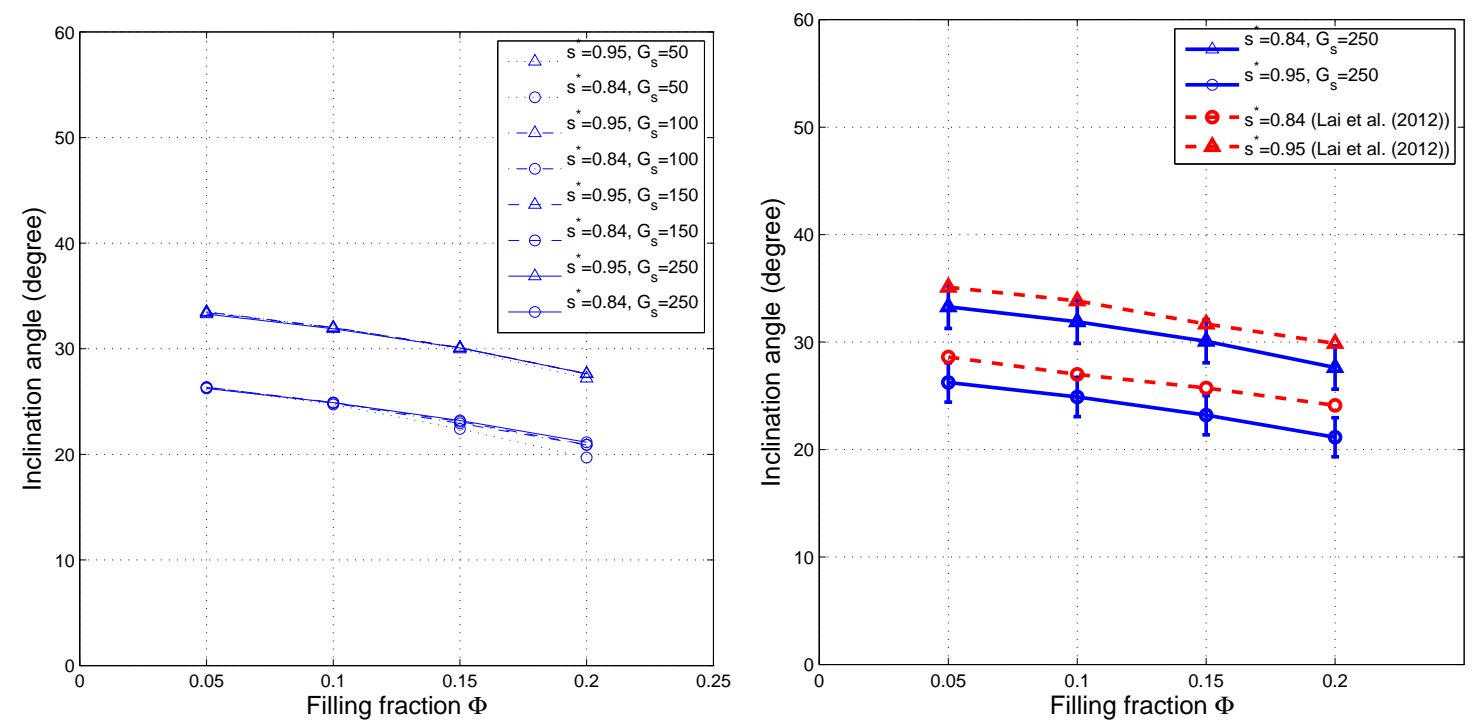

Figure 7: The averaged inclination angles of a compound vesicle with different filling ratio at different shear rate (left) and a comparison with the results obtained in [22] (right).

Section 2.2, the vesicles considered in this article are inextensible in our simulations. The filling fractions inside the vesicle (i.e., the ratio of the area of the particle and that of the region enclosed by the vesicle) are $\phi=0.05,0.1,0.15$ and 0.2 . The mass centers of both vesicle and freely moving neutrally buoyant particle are located at $(10,10)$ initially. The densities of both fluid and particle are $1 \mathrm{~g} / \mathrm{cm}^{3}$. The viscosity is $\mu_{f}=1 \mathrm{~g} /(\mathrm{cm} \mathrm{s})$ inside and outside the RBC membrane. The grid resolution for the computational domain is 80 grid points per $10 \mu \mathrm{m}$. The time step $\Delta t$ is $5 \times 10^{-4} \mathrm{~ms}$. Several different shear rates from $50 / \mathrm{s}$ to $250 / \mathrm{s}$ have been considered. The initial velocity field is a simple shear flow associated with the given shear rate without particle. Snapshots of velocity field next to the compound vesicle with different filling fractions at the shear rate $G_{s}=250 / \mathrm{s}$ are shown in Figure 6 in which the vesicle shows a typical tank-treading motion with a fixed inclination angle. The comparisons of the averaged inclination angle of the compound vesicle with different filling ratio are presented in Figure 7. For the larger shear rates, inclination angles are about the same. Our results of inclination angle are consistent with those obtained in [22]. The error bars in Figure 7 represent the ranges of inclination angle which is obtained from the farthest one among the membrane points to the mass center of the vesicle. Such angle varies slightly while the membrane tank-treads due to the use of a polygonal shape from a spring network to model vesicle membrane (see Figure 2). The observed decrease of the inclination angle, when increasing the filling fraction, is due to the increase of the effective viscosity inside the vesicle membrane; this has 


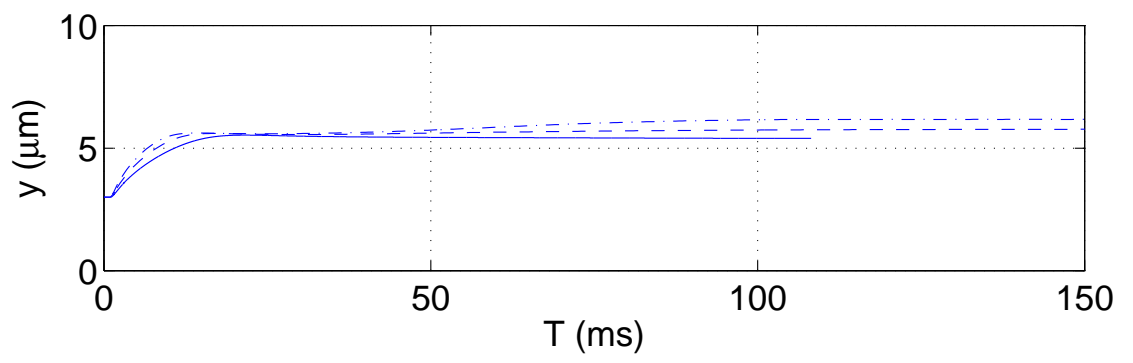

Figure 8: Histories of the mass center of the neutrally buoyant particle inside a vesicle: $\phi=0.2$ (dash-dotted line), 0.3 (dashed line) and 0.4 (solid line).

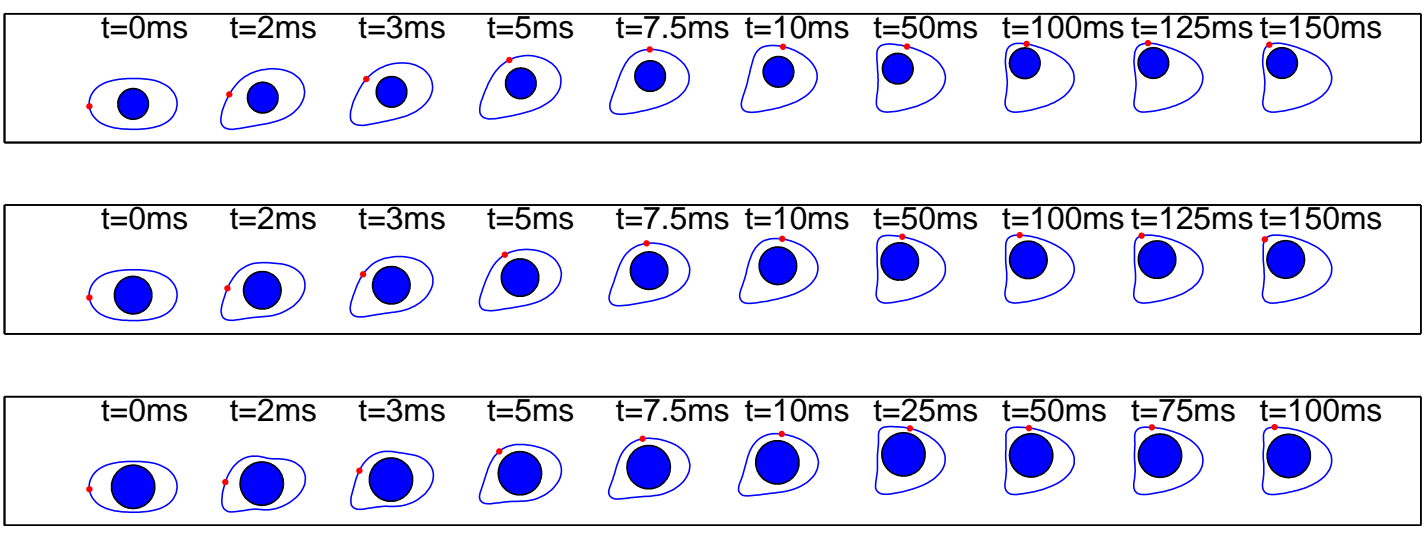

Figure 9: The positions of a compound vesicle during its lateral migration: $\phi=0.2$ (top), 0.3 (middle) and 0.4 (bottom).

been discussed in, e.g., [34, 35]. For our cases, there are many empirical formulas to compute the effective viscosity of the mixture of particle and fluid inside a membrane (e.g., see [36] for details). When increasing the filling fraction to $34 \%$ (resp., 39\%), the compound vesicle of $s^{*}=0.84$ (resp., 0.95 ) tumbles at the shear rate $G_{s}=250 / \mathrm{s}$.

Next we have considered the cases of a compound vesicle in a Poiseuille flow. We have kept the same parameters for the simulations except the following ones: The computational domain is $\Omega=80 \times 10 \mu \mathrm{m}^{2}$; the swelling ratio is $s^{*}=0.9$, the filling fractions are $\phi=0.2,0.3$ and 0.4 , and the viscosity $\mu_{f}=1 \mathrm{~g} /(\mathrm{cm} \mathrm{s})$. For driving the Poiseuille flow, we have applied the pressure gradient in the horizontal direction so that the maximum speed at the central line is $1 \mathrm{~cm} / \mathrm{s}$ when having no compound vesicle. The compound vesicle is placed next to the bottom wall with the mass center $3 \mu \mathrm{m}$ above the wall and the inclination angle of zero degree (see Figure 9). Due to the combined effects of the vesicle deformability and the shear gradient of the Poiseuille flow, the compound vesicle migrates toward an equilibrium position at the 

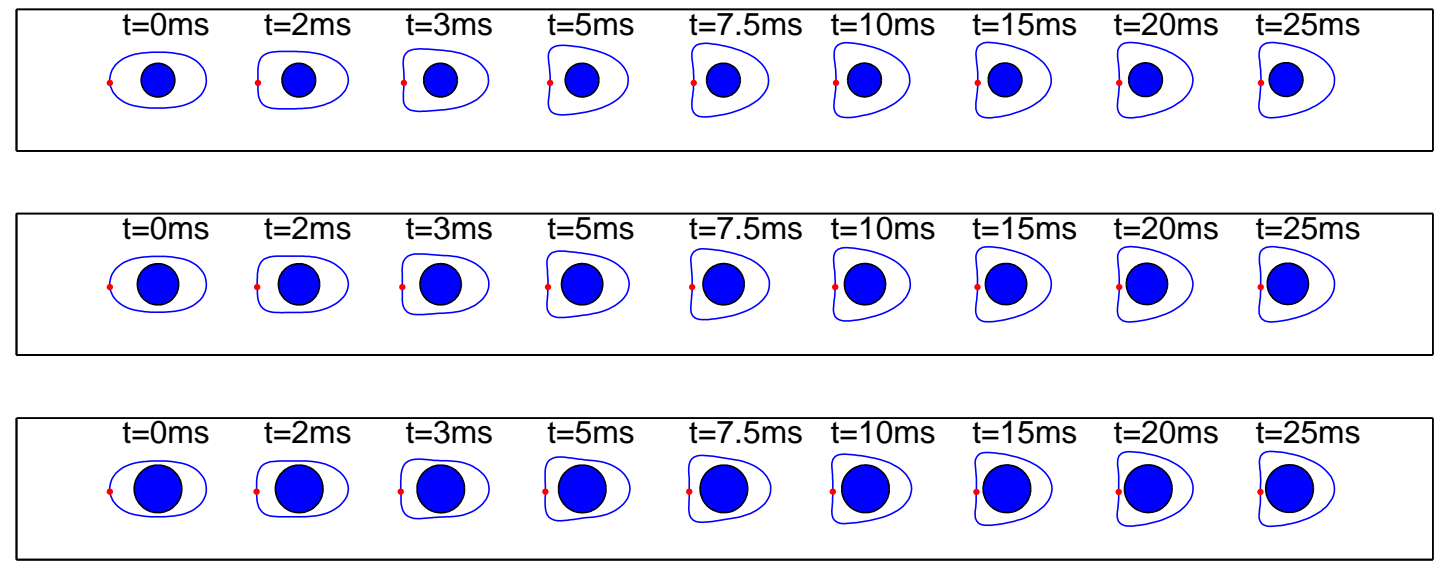

Figure 10: The positions of a compound vesicle initially located at the middle of the channel: $\phi=0.2$ (top), 0.3 (middle) and 0.4 (bottom).

central region of the channel similar to the ones without particle inside. The histories of the mass center of the neutrally buoyant particle for different filling fractions are presented in Figure 8. The positions of a compound vesicle during its lateral migration are shown in Figure 9 for $\phi=0.2,0.3$ and 0.4. Both figures show that the neutrally buoyant particle migrates due to the push from the motion of the vesicle membrane since such particle can not move across streamlines under creeping flow condition. After the vesicle reaches the central region of the channel, the particle moves in the horizontal direction as it should be. When placing the same compound vesicle at the middle of the channel initially, it moves in the horizontal direction and the membrane evolves into a parachute shape as presented in Figure 10. Both results show that the motion of compound vesicle is dominated by the motion of the vesicle membrane. The last case we have considered is the interaction of a compound vesicle with the swelling ratio is $s^{*}=0.9$ and filling fraction $\phi=0.4$ interacting with seven vesicles of $s^{*}=0.481$ (modeled as healthy RBCs). The others parameters are the same as above two cases except that the computational domain is $\Omega=40 \times 10 \mu \mathrm{m}^{2}$. All vesicles, including the compound one, migrate to the central region of the channel and stay there after reaching the central region as shown in Figure 11. In this section, these numerical results involving a compound vesicle in Poiseuille flow under the creeping flow condition suggest that such condition is not a right one since iRBCs move closer to the walls as observed in [37]. The motion of a compound vesicle at the finite Reynolds number will be presented in a forthcoming article. 

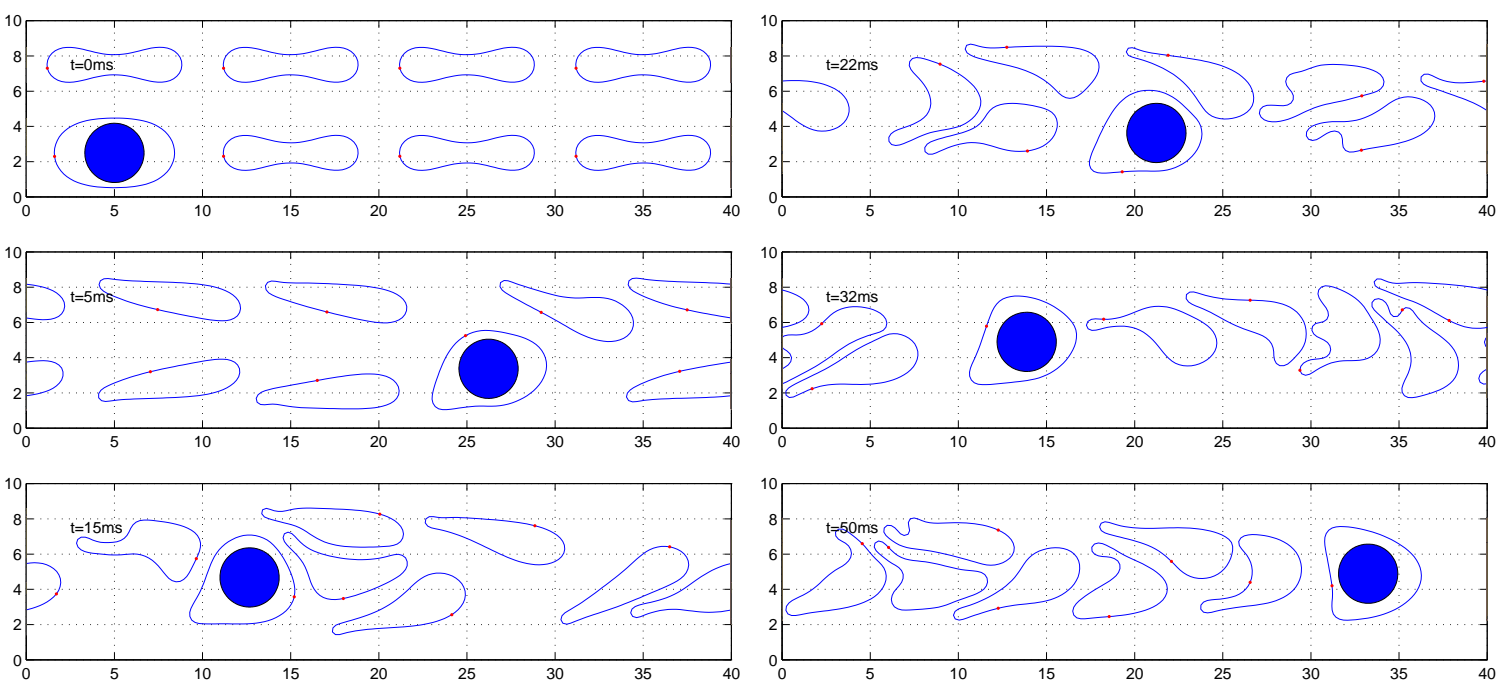

Figure 11: The positions of a compound vesicle with filling fraction $\phi=0.4$ interacting with seven vesicles modeled as healthy RBCs in a microchannel at $\mathrm{t}=0,5,15,22,32$, $50 \mathrm{~ms}$ (from top to bottom and from left to right).

\section{Conclusions}

In this article we first present a new distributed Lagrange multiplier/fictitious domain method for simulating fluid-particle interaction in Stokes flow. A conjugate gradient method driven by both pressure and distributed Lagrange multiplier, called a oneshot method, has been developed to solve the discrete Stokes problem while enforcing the rigid body motion within the region occupied by the particle. We validate the methodology by comparing the numerical results of a neutrally buoyant particle of either a circular or elliptic shape with the associated Jeffery's solutions. We then successively combine the above methodology with an immersed boundary method and an elastic membrane modeled by a spring network to simulate the dynamics of a compound vesicle under creeping flow condition. In shear flow, the inclination angles of the compound vesicle with different filling fractions are consistent with those obtained in the literature. At larger filling fractions, the compound vesicle tumble since the effective viscosity of the mixture of fluid and particle is high enough. In Poiseuille flow, the motion of compound vesicle is dominated by the motion of the vesicle membrane. After the compound vesicle migrates from the initial position next the wall to the central region of the channel, the particle moves horizontally without crossing the streamlines, which is what we expect about the behavior of a neutrally buoyant particle in Stokes flow. The numerical results involving a compound vesicle in Poiseuille flow under the creeping flow condition suggest that such condition is not a right one since iRBCs move closer to the walls as observed in [37]. The motion of 
a compound vesicle at the finite Reynolds number will be presented in a forthcoming article.

\section{Acknowledgments}

This work is supported by NSF Grants (DMS-0914788 and DMS-1418308). We acknowledge the helpful comments of James Feng, Ming-Chih Lai and Sheldon X. Wang.

\section{References}

[1] G. W. Schmid-Schonbein, Y. Y. Shih, and S. Chien, Morphometry of human leukocytes, Blood 56 (1980), 866-875.

[2] M. Diez-Silva, M. Dao, J. Han, C. T. Lim, and S. Suresh, Shape and Biomechanical Characteristics of Human Red Blood Cells in Health and Disease, MRS Bull. 35 (2010), 382-388.

[3] F. K. Glenister, R. L. Coppel, A. F. Cowman, N. Mohandas, and B. M. Cooke, Contribution of parasite proteins to altered mechanical properties of malariainfected red blood cells, Blood 99 (2002), 1060-1063.

[4] S. K. Veerapaneni, Y.-N. Young, P. M. Vlahovska, and J. Blawzdziewicz, Dynamics of a compound vesicle in shear flow, Phys. Rev. Lett. 106 (2011), 158103.

[5] B. Kaoui, T. Krüger, and J. Harting, Complex dynamics of a bilamellar vesicle as a simple model for leukocytes, Soft Matter 9 (2013), 8057-8061.

[6] G. B. Nash, E. O'Brien, E. C. Gordon-Smith, and J. A. Dormandy, Abnormalities in the mechanical properties of red blood cells caused by Plasmodium falciparum, Blood 74 (1989), 855-861.

[7] Y. Imai, H. Kondo, T. Ishikawa, C. T. Lim, T. Yamaguchi, Modeling of hemodynamics arising from malaria infection, J. Biomech. 43 (2010), 1386-1393.

[8] T. Wu and J. J. Feng, Simulation of malaria-infected red blood cells in microfluidic channels: Passage and blockage, Biomicrofluidics 7 (2013), 044115.

[9] L. Shi, T.-W. Pan, R. Glowinski, Deformation of a single blood cell in bounded Poiseuille flows, Phys. Rev. E 85 (2012), 016307. 
[10] L. Shi, T.-W. Pan, R. Glowinski, Lateral migration and equilibrium shape and position of a single red blood cell in bounded Poiseuille flows, Phys. Rev. E 86 (2012), 056308.

[11] L. Shi, T.-W. Pan, R. Glowinski, Numerical simulation of lateral migration of red blood cells in Poiseuille flows, Int. J. Numer. Methods Fluids 68 (2012), 1393-1408.

[12] R. Glowinski, T.-W. Pan, T. Hesla, D. D. Joseph, A distributed Lagrange multiplier/fictitious domain method for particulate flows, Int. J. Multiphase Flow 25 (1999), 755-794.

[13] R. Glowinski, T.-W. Pan, T. Hesla, D. D. Joseph, J. Périaux, A fictitious domain approach to the direct numerical simulation of incompressible viscous flow past moving rigid bodies: Application to particulate flow. J. Comput. Phys. 169 (2001), 363-427.

[14] T.-W. Pan, D. D. Joseph, R. Glowinski, Modeling Rayleigh-Taylor instability of a sedimenting suspension of several thousand circular particles in direct numerical simulation, J. Fluid Mech. 434 (2001), 23-37.

[15] T.-W. Pan, R. Glowinski. Direct simulation of the motion of neutrally buoyant circular cylinders in plane Poiseuille flow. J. Comput. Phys. 181 (2002), 260279 .

[16] T.-W. Pan, D. D. Joseph, D. Bai, R. Glowinski, D. Sarin, Fluidization of 1204 spheres: simulation and experiments. J. Fluid Mech. 451 (2002), 169-191.

[17] T.-W. Pan, R. Glowinski, Direct simulation of the motion of neutrally buoyant balls in a three-dimensional Poiseuille flow. C. R. Mecanique, Acad. Sci. Paris 333 (2005), 884-895.

[18] B. H. Yang, J. Wang, D. D. Joseph, H. H. Hu, T.-W. Pan, R. Glowinski, Numerical study of particle migration in tube and plane Poiseuille flows. J. Fluid Mech. 540 (2005), 109-131.

[19] T.-W. Pan, C.-C. Chang, R. Glowinski, On the motion of a neutrally buoyant ellipsoid in a three-dimensional Poiseuille flow. Comput. Methods Appl. Mech. Engrg. 197 (2008), 2198-2209.

[20] T.-W. Pan, S.-L. Huang, S.-D. Chen, C.-C. Chu, C.-C. Chang, A numerical study of the motion of a neutrally buoyant cylinder in two dimensional shear flow, Computers \& Fluids 87 (2013), 57-66.

[21] N. Sharma, Y. Chen, N. A. Patankar, A distributed lagrange multiplier based computational method for the simulation of particulate-Stokes flow, Comput. Methods Appl. Mech. Engrg. 194 (2005), 4716-4730. 
[22] M.-C. Lai, W.F. Hu, W.-W. Lin, A fractional step immersed boundary method for stokes flow with an inextensible interface enclosing a solid particle, SIAM J. Sci. Comput. 34 (2012), B692-B710.

[23] K. Tsubota, S. Wada, Effect of the natural state of an elastic cellular membrane on tank-treading and tumbling motions of a single red blood cell, Phys. Rev. E 81 (2010), 011910.

[24] A. Alexeev, R. Verberg, A. C. Balazs, Modeling the interactions between deformable capsules rolling on a compliant surface, Soft Matter 2 (2006), 499-509.

[25] M.O. Bristeau, R. Glowinski, and J. Périaux, Numerical methods for the Navier-Stokes equations. Applications to the simulation of compressible and incompressible viscous flow, Computer Physics Reports 6 (1987), 73-187.

[26] R. Glowinski, Finite element methods for incompressible viscous flow, in: P.G. Ciarlet, J.L. Lions (Eds.), Handbook of Numerical Analysis, vol. IX, NorthHolland, Amsterdam, 2003, pp. 3-1176.

[27] C.S. Peskin, Numerical analysis of blood flow in the heart, J. Comput. Phys. 25 (1977), 220-252.

[28] Peskin CS, McQueen DM. Modeling prosthetic heart valves for numerical analysis of blood flow in the heart. J. Comput. Phys. 37 (1980), 11332.

[29] C.S. Peskin, The immersed boundary method, Acta Numer. 11 (2002), 479517.

[30] J. Chorin, T. J. R. Hughes, M. F. McCracken, J. E. Marsden, Product formulas and numerical algorithms, Commun. Pure Appl. Math. 31 (1978), 205-256.

[31] R. Glowinski, T.-W. Pan, J. Périaux, A one shot domain decomposition/fictitious domain method for the Navier-Stokes equations, in: D.E. Keyes and J. Xu (Eds.), Domain Decomposition Methods in Scientific and Engineering Computing, AMS, Providence, RI, 1994, pp. 211-222.

[32] R. Glowinski, T.-W. Pan, J. Périaux, A one shot domain decomposition/fictitious domain method for the solutions of elliptic equations, in: A. Ecer, J. Hauser, P. Leca and J. Périaux (Eds.), Parallel Computational Fluid Dynamics: New Trends and Advances, North-Holland, Amsterdam, 1995, pp. 317-324.

[33] G. B. Jeffery, The motion of ellipsoidal particles immersed in a viscous fluid, Proc. R. Soc. Lond. A 102 (1922), 161-79.

[34] J. Beaucourt, F. Rioual, T. Séon, T. Biben, and C. Misbah, Steady to unsteady dynamics of a vesicle in a flow, Phys. Rev. E 69 (2004), 011906. 
[35] S. R. Keller, R. Skalak, Motion of a tank-treading ellipsoidal particle in a shear flow, J. Fluid Mech. 120 (1982), 27-47.

[36] N.-S. Cheng, A. W.-K. Law, Exponential formula for computing effective viscosity, Powder Technology 129 (2003), 156-160.

[37] H. W. Hou, A. A. S. Bhagat, A. G. L. Chong, P. Mao, K. S. W. Tan, J. Han, C. T. Lim, Deformability based cell margination-A simple microfluidic design for malaria-infected erythrocyte separation, Lab Chip 10 (2010), 2605-2613. 